\title{
ANESTHETIC CHALLENGES IN AN ADOLESCENT WITH CONGENITAL BILATERAL TIBIA-FIBULA AMELIA WITH HIP DYSPLASIA FOR HERNIA REPAIR
}

\author{
Dr Madhura Maiya'1, Dr. Nita D'Souza² \\ 1. Apollo Hospital, Bangalore \\ 2. Ruby Hall Clinic, Pune
}

\section{BACKGROUND AND AIM}

Undiagnosed mesodermal defects presenting with bilateral tibia and fibula amelia, single kidney, hip dysplasia and severe kyphoscoliosis in an adolescent with abdominal muscle weakness causing femoral hernia is an anesthetic challenge.

Our patient had all clinical features of Fibular Amelia, Tibial Campomelia and Oligosyndactyl (FATCO) Syndrome ${ }^{1,2}$ but no genetic analysis was done (fig1). FATCO Syndrome is an extremely rare disorder with limited literature of patient surviving up to adolescence. Multiple mesodermal defects with distorted anatomy, restrictive lung disease and severe kyphoscoliosis, posed challenges for planning anesthesia.

\section{METHODS}

An 18 year old girl, weighing $20 \mathrm{~kg}$, height $80 \mathrm{~cm}$, presented with left femoral hernia. Since birth she had bilateral tibial and fibular amelia, hip dysplasia, absent left kidney, external ear malformation, polydactyly and syndactyly (fig 2). On presentation she had severe kyphoscoliosis and mild restrictive lung disease with distorted anatomy of hip and surgical site (fig3). We avoided central neuraxial block in view of severe kyphoscoliosis, absence of lower limb with hip dysplasia and short stature. We planned general anesthesia and ultrasound guided Transversus Abdominal Plane (TAP) block. Standard monitoring was done. Patient was induced with Inj. Fentanyl 40mcg; Inj. Propofol $80 \mathrm{mg}$ using supreme LMA no. 3. Muscle relaxant was avoided.

\section{RESULTS}

After induction of general anesthesia; Ultrasound guided Transversus Abdominal Plane (TAP) block was given with $0.25 \%$ Ropivacaine $20 \mathrm{ml}$ on left side (fig4). Despite the anterior superior iliac spine being placed laterally and small in size, the internal oblique, external oblique and transversus abdominis appeared as usual, though all with similar thickness. Internal oblique was not larger than other two muscles. This could be attributed to her inability to walk and hip dysplasia. Maintenance of anesthesia with sevoflurane $1.5-2 \%, 50 \%$ oxygen and air was done. Procedure was uneventful.Patient was comfortable with postoperative analgesia lasting for 20 hours.

\section{CONCLUSION}

Undiagnosed malformation syndrome put forth multisystem management challenges. Ultrasound guided block are a suitable aid in cases with malformation and distorted anatomy to facilitate surgical and postoperative analgesia with no use of NSAIDS and opioids.

References:

1. Kitaoka T, Namba N, Kim JY, Kubota T, Miura K, Miyoshi Y, et al. A Japanese male patient with fibular aplasia, tibial campomelia and oligodactyl : an additional case report. Clin Pediatr Endocrinol 2009;18(3):81-6.

2. Goyal N, Kaur R, Gupta M, Bhatty S, Paul R, et al. FATCO Syndrome Variant -Fibular Hypoplasia, Tibial Campomelia and Oligosyndactyl_A Case Report. J Clin Diagn Res. 2014 Sep;8(9): LD01-2 .

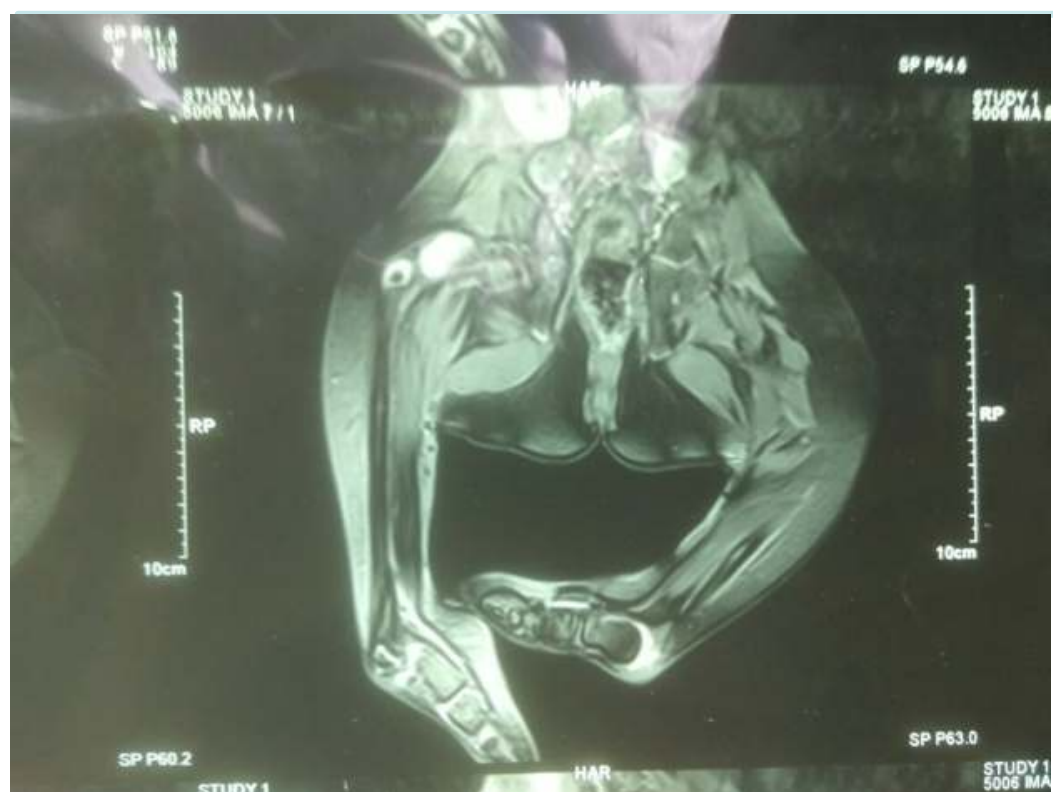

Fig1 FATCO Anomaly

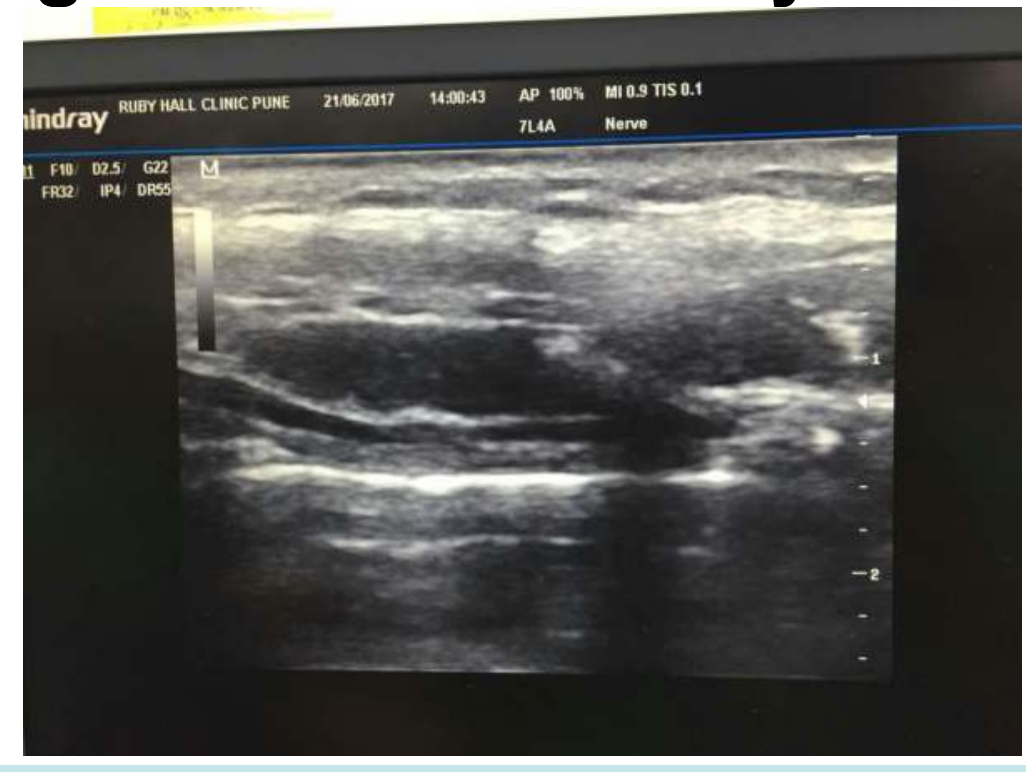

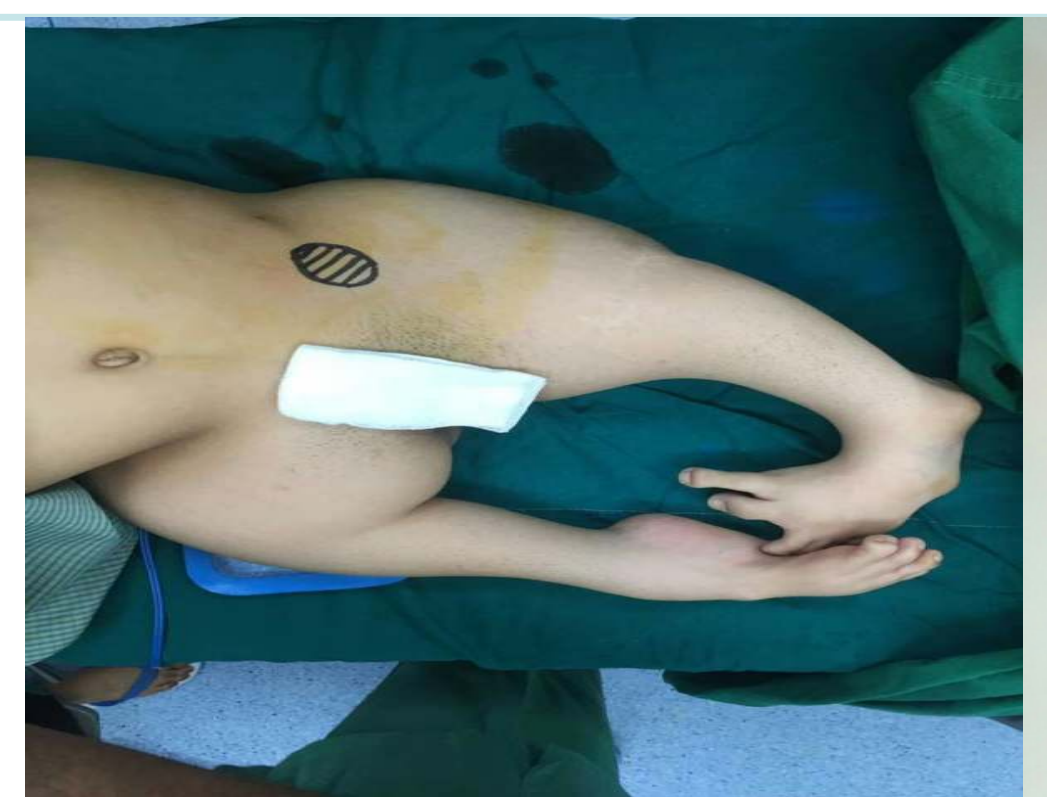

Fig 2 Clinical presentation Fig 3 Kyphoscoliosis 